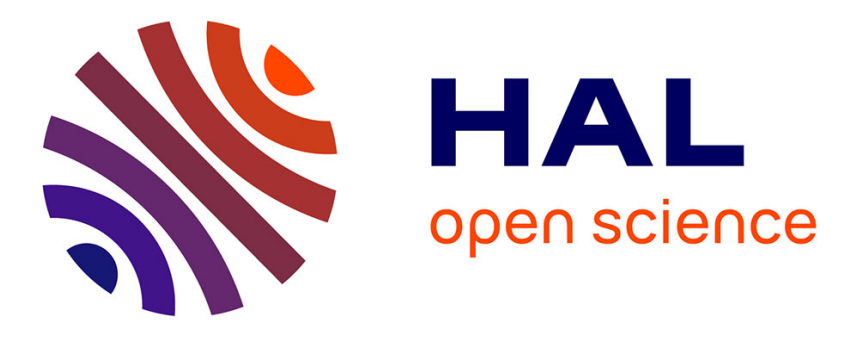

\title{
On a Class of Stochastic Multilayer Networks
}

Bo Jiang, Philippe Nain, Don Towsley, Saikat Guha

\section{To cite this version:}

Bo Jiang, Philippe Nain, Don Towsley, Saikat Guha. On a Class of Stochastic Multilayer Networks. [Research Report] Inria Grenoble Rhône-Alpes, Université de Grenoble; Dante. 2018. hal-01669368v1

\section{HAL Id: hal-01669368 https://hal.inria.fr/hal-01669368v1}

Submitted on 20 Dec 2017 (v1), last revised 2 Feb 2018 (v2)

HAL is a multi-disciplinary open access archive for the deposit and dissemination of scientific research documents, whether they are published or not. The documents may come from teaching and research institutions in France or abroad, or from public or private research centers.
L'archive ouverte pluridisciplinaire HAL, est destinée au dépôt et à la diffusion de documents scientifiques de niveau recherche, publiés ou non, émanant des établissements d'enseignement et de recherche français ou étrangers, des laboratoires publics ou privés. 


\section{On a Class of Stochastic Multilayer Networks}

\author{
Bo Jiang \\ University of Massachusetts \\ Amherst, MA 01003, USA \\ bjiang@cs.umass.edu \\ Don Towsley \\ University of Massachusetts \\ Amherst, MA 01003, USA \\ towsley@cs.umass.edu
}

\author{
Philippe Nain \\ Inria \\ Ecole Normale Supérieure de Lyon, LIP, 46 allée d'Italie \\ 69364 Lyon, France \\ philippe.nain@inria.fr \\ Saikat Guha \\ University of Arizona \\ Tucson, AZ 85721, USA \\ saikat@optics.arizona.edu
}

\begin{abstract}
In this paper, we introduce a new class of stochastic multilayer networks. A stochastic multilayer network is the aggregation of $M$ networks (one per layer) where each is a subgraph of a foundational network $G$. Each layer network is the result of probabilistically removing links and nodes from $G$. The resulting network includes any link that appears in at least $K$ layers. This model is an instance of a non-standard site-bond percolation model. Two sets of results are obtained: first, we derive the probability distribution that the $M$ layer network is in a given configuration for some particular graph structures (explicit results are provided for a line, an algorithm is provided for a tree), where a configuration is the collective state of all links (each either active or inactive). Next, we show that for appropriate scalings of the node and link selection processes in a layer, links are asymptotically independent as the number of layers goes to infinity, and follow a Poisson distribution. Numerical results are provided to highlight the impact of having several layers on some metrics of interest (including expected size of the cluster a node belongs to in the case of the line). This model finds applications in wireless communication networks with multichannel radios, multiple social networks with overlapping memberships, transportation networks, and, more generally, in any scenario where a common set of nodes can be linked via co-existing means of connectivity.
\end{abstract}

\section{CCS CONCEPTS}

-Mathematics of computing $\rightarrow$ Random graphs; Paths and connectivity problems; •Networks $\rightarrow$ Network structure;

\section{KEYWORDS}

Stochastic multilayer network, percolation

\section{ACM Reference format:}

Bo Jiang, Philippe Nain, Don Towsley, and Saikat Guha. 2018. On a Class of Stochastic Multilayer Networks. In Proceedings of ACM SIGMETRICS conference, Irvine, California, USA, fune 2018 (SIGMETRICS'18), 13 pages. DOI:

Permission to make digital or hard copies of part or all of this work for personal or classroom use is granted without fee provided that copies are not made or distributed for profit or commercial advantage and that copies bear this notice and the full citation on the first page. Copyrights for third-party components of this work must be honored. For all other uses, contact the owner/author(s).

SIGMETRICS'18, Irvine, California, USA

(c) 2018 Copyright held by the owner/author(s). ..\$15.00

DOI:

\section{INTRODUCTION}

There is an increasing need to understand how different networks interact with each other. One means of such interaction arises when users (nodes) belong to two or more networks (layers). In recent years, there has been a surge of interest in such multilayer networks $[8,20]$ due to their relevance in problems stemming in varied fields such as multifrequency wireless communication networks [32], multiple online social networks serving a common population [5, 23, 37] just to name a few. Various models of multilayer networks (also termed multiplex networks and composite networks in the literature) relevant to different application scenarios have been proposed, in particular stochastic multilayer networks whose constructions can be described by one or more control parameters (such as probability of the presence of a node, edge or more complex attributes). For such networks, a wide variety of percolation formulations have been proposed and studied, e.g., competition between layers [40], weak percolation [7], $k$-core percolation [3], directed percolation [2], spanning connectivity of a multilayer sitepercolated network [1], and bond percolation [17]. However, even simple multilayer network models have proven extremely difficult to solve exactly [1], because of which most of this aforesaid recent literature on properties of multilayer networks consists of numerical and heuristic analyses.

Our goal in this paper is to consider a simple model for a stochastic multilayer network and to attempt exact characterization of the joint probability distribution of the collective (on-off) configuration of the links of the multilayer network. We provide exact results and efficient algorithms for some special graphs, and prove some complexity-theoretic hardness results in the general case. Our model is as follows. A multilayer network consists of $M$ co-existing networks $G^{(1)}, G^{(2)}, \ldots, G^{(M)}$ connecting a common set of users. Each user is active in only a subset of these networks. Consequently, a user active in both $G^{(1)}$ and $G^{(2)}$ can help connect two other users that are active in $G^{(1)}$ alone, and in $G^{(2)}$ alone, respectively, by forming a bridge. Figure 1 illustrates an example with $M=3$ networks (layers), where a path connecting $v_{1}$ and $v_{2}$ must traverse all three layers, and one such path is shown to go through the bridge nodes $v_{3}$ and $v_{4}$, both of which belong to more than one layer. A stochastic multilayer network is a graph $G=(V, E)$ along with a random process by which each network layer is obtained from $G$ by randomly removing links (called link thinning) and randomly deactivating nodes, and a process by which the $M$ thinned layers 
are merged into a single graph. Layer $m, 1 \leq m \leq M$ is a subgraph of $G$ consisting of all remaining active nodes and all links between active nodes not removed through link thinning. There are different ways of creating a multilayer network out of the $M$ layers. One is simply to take the union of (the nodes and links of) all the layer graphs; this is illustrated by the three layer network in Figure 1. We consider a slightly more general process whereby all active nodes are included in the final graph and all links that appear in at least $K$ layers.

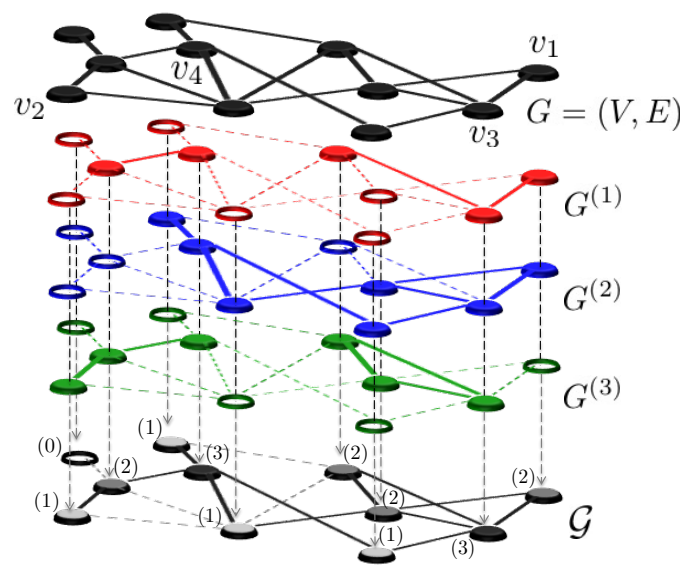

Figure 1: Multilayer network with three layers.

Concrete examples of such multilayer networks are: (1) a network of cities connected via different airline companies where each city is served only by a subset of all the airlines $[5,10],(2)$ a network of users with accounts on multiple online social networks [26], and (3) a military communication network of units equipped with radios that can listen and transmit simultaneously on a subset of multiple frequencies [33].

With a more liberal interpretation of "co-existence", such multilayer networks may also arise from taking snapshots of a single network at different time epochs. For example, consider a duty-cycled wireless sensor network where each sensor is active or dormant according to a random periodic schedule and each period is divided into $M$ slots. The $m$-th layer $G^{(m)}$ then consists of sensors that are active in the $m$-th slot. Duty-cycled models have been studied in the wireless sensor network literature (e.g., [4] and the reference therein), where the underlying networks are usually random geometric graphs and the focus is on the connectivity of each layer, which is a much stronger notion of connectivity than connectivity in our aggregate network.

Denote the configuration of a multilayer network by the collection of states of all links in the underlying graph $G$ after the layers have been merged. Here the state of each link is either active (1) or inactive (0). We are interested in characterizing the configuration probability distribution of the multilayer network under the assumption that thinning and deactivation operations occur as independent events. Such a characterization can be useful for computing quantities such as the distribution of the sizes of connected components and average path lengths. We show that in general, computing the network configuration distribution is hard - for example, in most cases computing the probability that there are no active links in the merged graph is \#P-hard. On the other hand we have partial positive results for some classes of graphs including trees. Moreover, we consider the behavior of this distribution in the limit as $M \rightarrow \infty$. Our contributions are:

- We present a new model of a stochastic multilayer network based on link thinning and node deactivation, and show that in general it is a difficult problem to compute the probabilities of the multilayer network configurations and it remains difficult even to approximate these probabilities.

- We develop efficient algorithms for computing multilayer network configuration probabilities for line and tree topologies.

- We consider a setting where the number of layers $M$ goes to infinity and where the link thinning probabilities and node deactivation probabilities are functions of $M$. We provide conditions for when link existence events are asymptotically independent.

The paper follows the following organization. Section 2 presents our stochastic multilayer model. The hardness of the problem of computing the multilayer network configuration probabilities is addressed in Section 3. Exact results and efficient computational algorithms are presented in Section 4 and the asymptotic independence of the link states as $M \rightarrow \infty$ is found in Section 5. A discussion of related work can be found in Section 6 and conclusions are drawn in Section 7.

\section{MODEL}

Let $G=(V, E)$ denote the underlying connectivity network, where $V$ is the set of nodes and $E \subset V \times V$ is the set of links that represent all possible connections between pairs of nodes in $V$ (in the graph/percolation community a node is called a vertex/site and a link is called an edge/a bond; throughout we will use node and link which are commonly used in communication networks). We assume network $G$ is connected.

Consider an $M$-layer network whose layers are sub-networks of $G$ obtained by randomly removing links (called link thinning) and deactivating nodes. When a node is deactivated on a layer, all links incident on it are removed from the same layer, including those that have survived the independent link thinning process. More precisely, the $M$-layer network is obtained from $G$ as follows. Let $\mathcal{M}=\{1,2, \ldots, M\}$ be the index set for layers. Let $Y_{\mathcal{M}, E}=$ $\left\{Y_{m, \ell}: \ell \in E, m \in \mathcal{M}\right\}$ and $Z_{\mathcal{M}, V}=\left\{Z_{m, i}: i \in V, m \in \mathcal{M}\right\}$ be two mutually independent sets of independent Bernoulli random variables. For the $m$-th layer $G^{(m)}=\left(V, E^{(m)}\right)$, node $i$ is active if and only if $Z_{m, i}=1$, and the link set $E^{(m)}$ is given by $E^{(m)}=$ $\left\{\ell \in E: W_{m, \ell}=1\right\}$, where $W_{m, \ell}=Y_{m,(i, j)} Z_{m, i} Z_{m, j}$ for link $\ell=(i, j)$. Note that link $\ell=(i, j)$ is in $E^{(m)}$ if and only if it is not thinned $\left(Y_{m, \ell}=1\right)$ and both endpoints $i, j$ are active on the $m$-th layer $\left(Z_{m, i}=Z_{m, j}=1\right)$. We assume that the link thinning probabilities and the node activation probabilities are the same across different layers but may depend on individual links and nodes, i.e. $p_{\ell}=\mathbb{E}\left[Y_{m, \ell}\right]$ and $q_{i}=\mathbb{E}\left[Z_{m, i}\right]$ for all $m \in \mathcal{M}, \ell \in E$ and $i \in V$. This assumption will be relaxed in Section 5.3. We also assume that all $p_{\ell}$ 's and $q_{i}$ 's are strictly positive. 
Let $W_{\ell} \in \mathbb{N}$ denote the number of layers in which link $\ell$ is present. Formally, for $\ell=(i, j)$,

$$
W_{\ell}=\sum_{m \in \mathcal{M}} W_{m, \ell}
$$

Note that we have suppressed the explicit dependence on $M$ of all random variables for notational simplicity. No confusion should arise. Given some threshold $K \in \mathbb{N}$, let $X_{\ell}=\mathbb{1}_{\left\{W_{\ell} \geq K\right\}}$, where $\mathbb{1}_{A}$ is the indicator of event $A$. We say that link $\ell$ is active (inactive) in the multilayer network if $X_{\ell}=1\left(X_{\ell}=0\right)$. We obtain a merged network $\mathcal{G}=(V, \mathcal{E})$, where $\mathcal{E}=\left\{\ell \in E: X_{\ell}=1\right\}$ is the set of active links; we say the multilayer network has link configuration $\mathcal{E}$, or equivalently, configuration $X_{E} \triangleq\left\{X_{\ell}: \ell \in E\right\}$. We will use the terms configuration and state interchangeably. The parameter $K$ determines the robustness of links in $\mathcal{G}$; a larger value of $K$ results in more robust links but a possibly less well connected network $\mathcal{G}$. Note that when $K=1, \mathcal{G}$ is simply the union of the layers, i.e. $\mathcal{G}=\bigcup_{m=1}^{M} G^{(m)}=\left(V, \bigcup_{m=1}^{M} E^{(m)}\right)$. More generally, we call any vector $x$ with component $x_{\ell} \in\{0,1\}$ for $\ell \in E$ a link configuration of the multilayer network, and we call it a feasible link configuration if $\mathbb{P}\left[X_{\ell}=x_{\ell}, \forall \ell \in E\right]>0$.

Figure 1 shows a three-layer network with $p_{\ell}=1$ for all $\ell$ (i.e. no link is thinned) and $K=1$. Inactive links on each layer and in the merged network at the bottom are represented by dashed lines. The bottom graph is $\mathcal{G}=\bigcup_{m=1}^{3} G^{(m)}$. In this network, node $v_{1}$ belongs to two layers, $v_{2}$ belongs to three layers and these nodes have two layers in common, and $v_{3}$ belongs to one layer.

\section{HARDNESS RESULTS}

In this section, we show that it is very hard to compute the probability of given link configurations in arbitrary multilayer networks. We show in Section 3.1 that one source of hardness is the generality of the underlying connectivity network $G$. On the other hand, we show in Section 3.2 that hardness may arise from the multilayer nature of the problem, even when the underlying network $G$ has a simple structure such as a clique, which makes the single layer problem easy. We assume $K=1$ throughout this section.

\subsection{Hardness for Single Layer General Graphs}

In this section, we show that it is hard to compute link configuration probabilities for general underlying connectivity network $G$ even when there is only one layer, i.e. $M=1$. The proof uses a reduction from the \#INDEPENDENT SET problem. Recall that an independent set is a set of vertices in a graph, no two of which are adjacent.

Definition 3.1 (\#INDEPENDENT SET). Given a graph G, count the number of independent sets in $G$.

Corollary 4.2 of [38] shows that many special cases of \#INDEPENDENT SET is \#P-complete, and hence the following

Lemma 3.2 ([38]). \#Independent Set is \#P-hard.

Recall that \#P is the class of counting problems that correspond to the decision problems in the class NP; while a decision problem asks whether there exists a solution, the corresponding counting problem asks how many solutions there are. Note that \#P-complete problems are NP-hard. The following lemma of [35] shows that \#INDEPENDENT SET is hard even to approximate.
Lemma 3.3 (Lemma A.3 of [35]). For any $\epsilon>0$, approximating the number of independent sets of a graph on $n$ vertices within $2^{n^{1-\epsilon}}$ is NP-hard.

Now we show that it is hard to compute the probability of the configuration where no link is active, even when there is no link thinning and the probability for a node to be active is $1 / 2$ for all nodes. It follows that the general case is also hard.

Proposition 3.4. Suppose $M=1$, $p_{\ell}=1$ for all $\ell \in E$, and $q_{i}=1 / 2$ for all $i \in V$. It is \#P-hard to compute the probability that the network is in the configuration with no active link. It is NP-hard to approximate this probability within a multiplicative factor of $2^{n^{1-\epsilon}}$ for any $\epsilon>0$, where $n=|V|$ is the number of nodes.

Proof. Given a graph $G=(V, E)$ and any node configuration $a: V \rightarrow\{0,1\}$, let $s(a)=a^{-1}(1)$ be the set of active nodes. Let $A$ denote the set of node configurations that results in an empty link set $\mathcal{E}$. Note that $a \in A$ if and only if $s(a)$ is an independent set of $G$. Since $q=1 / 2$, all node configurations are equally likely and there are $2^{n}$ of them, so $|A|=2^{n} \mathbb{P}[A]$. Thus counting the number of independent sets in $G$ is equivalent to computing $\mathbb{P}[A]$, as one can be easily obtained from the other through rescaling. Since it is \#P-hard to compute $|A|$ by Lemma 3.2, it is \#P-hard to compute $\mathbb{P}[A]$. By Lemma 3.3 , it is NP-hard to approximate $\mathbb{P}[A]$ within a multiplicative factor of $2^{n^{1-\epsilon}}$.

\subsection{Hardness for Multilayer Cliques}

In this section, we show that hardness arises in yet another dimension. Consider the case that the underlying network $G$ is a clique. In this case, it is trivial to compute link configuration probabilities for a single layer ${ }^{1}$, but for a large number of layers, the problem becomes hard. In fact, it is hard even to test the feasibility of a configuration, which is a simpler problem, since a configuration is feasible if and only if its probability is nonzero. Consider the Multilayer Clique Configuration (MCC) problem defined below.

Definition 3.5 (MultilaYer CLIQUE CONFIGURATION). Given an $M$-layer network with $G$ being a clique and a link configuration $x$, decide whether $x$ is feasible. Denote an instance by $(x, M)$.

Given any link configuration $x$, let $G(x)$ be the subgraph induced by the active links in $x$, i.e. $G(x)=(V, E(x))$ where $E(x)=\{\ell \in E$ : $\left.x_{\ell}=1\right\}$. We have the following feasibility test.

Lemma 3.6. Suppose the underlying network $G$ is a clique. A link configuration $x$ is feasible if and only if the induced subgraph $G(x)$ is covered by at most $M$ cliques.

Proof. If $x$ is feasible, then $x=\bigvee_{m=1}^{M} x^{(m)}$, where $x^{(m)}$ is a feasible link configuration of the $m$-th layer, and $\vee$ is componentwise maximum. Since $G$ is a clique, so is the subgraph $G\left(x^{(m)}\right)$ induced by $x^{(m)}$, if it is not empty. Thus $G(x)=\bigcup_{m=1}^{M} G\left(x^{(m)}\right)$ is covered by at most $M$ cliques.

For the reverse direction, suppose $G(x)$ can be covered by $M^{\prime} \leq$ $M$ cliques $C_{1}, \ldots, C_{M^{\prime}}$. On the $m$-th layer $G^{(m)}$, set a node to be active if and only if it is in $C_{m}$, which is a node configuration with positive probability. The resulting link configuration of the $M$-layer clique is exactly $x$, so $x$ is feasible.

${ }^{1}$ In this case, a node is active if and only if it is the end point of an active link. 
The above proof shows that any instance of MCC with $M=1$ is easy; the configuration $x$ is feasible if and only if the graph $G(x)$ induced by the active links in $x$ is itself a clique. For $M \geq|E|=$ $n(n-1) / 2$, where $n=|V|$ is the number of nodes, $x$ is always feasible, since $G(x)$ can be covered by $|E|$ links, which are cliques of size 2. For the general case, however, we now show it is NPcomplete by reduction from the Cligue Edge Cover (CEC) problem, which is known to be NP-complete. Recall

Definition 3.7 (CLIQUE EDGE COVER). Given a graph $G$ and an integer $k$, decide whether all edges of $G$ can be covered by at most $k$ cliques in $G$. Denote an instance by $(G, k)$.

Lemma 3.8 (Theorem 8.1 of [29]). CEC is NP-complete.

We have the following

\section{Proposition 3.9. $M C C$ is NP-complete.}

Proof. MCC is clearly in NP. We show that it is NP-hard by reduction from CEC. Fix an instance $(G, k)$ of CEC. Consider the clique $C$ that has the same node set as $G$. Let $x$ be the link configuration of $C$ such that $x_{\ell}=1$ if and only if $\ell$ is a link in $G$, i.e. $G$ is the subgraph of $C$ induced by $x$. Now we obtain an instance $(x, k)$ of MCC. The conclusion then follows from Lemma 3.6 and Lemma 3.8.

\section{EXACT RESULTS}

In this section, we provide recursions for computing link configuration probabilities. In the case of trees and lines, the recursions can be turned into a pseudopolynomial algorithm. In Section 4.1, we discuss two different ways of doing recursions. We then consider line and tree networks in Sections 4.2 and 4.3, respectively.

\subsection{Two Different Ways for Recursion}

There are two natural ways to obtain recursions for link configuration probabilities of a multilayer network. One is to do recursion on the number of layers and the other on the number of nodes. We briefly discuss the former in the present section and leave the latter for Sections 4.2 and 4.3. We restrict our discussion to the case $K=1$ in this section.

Consider an $M$-layer network with a general underlying graph $G=(V, E)$. For $K=1$, the merged network is $\mathcal{G}=\bigcup_{m=1}^{M} G^{(m)}$. Now considered a network $\mathcal{G}^{(k)}$ obtained by merging only the first $k$ layers, i.e. $\mathcal{G}^{(k)}:=\bigcup_{m=1}^{k} G^{(m)}$, for $k=1,2, \ldots, M$. Recall that a link is active in the $\mathcal{G}^{(k)}$ if it is active in at least one layer $1, \ldots, k$. Let $Q_{k}(x)$ be the probability of the link configuration $x \in\{0,1\}^{|E|}$ in $\mathcal{G}^{(k)}$. We have the following recursion,

$$
Q_{k+1}(x)=\sum_{y \in Y(x)} Q_{k}(y) Q_{1}(x-y)
$$

for all $x \in\{0,1\}^{|E|}$ and $k=1 \ldots, M$, where $Y(x):=\left\{y \in\{0,1\}^{|E|}\right.$ : $\left.y \leq_{c} x\right\}, x \in\{0,1\}^{|E|}$, is the set of vectors in $\{0,1\}^{|E|}$ componentwise smaller than or equal to vector $x$.

For instance, if $|E|=3$ and $x=(0,1,1)$ then

$$
Y(x)=\{(0,1,1),(0,0,1),(0,1,0),(0,0,0)\} .
$$

Note that for any vectors $x, y \in\{0,1\}^{|E|}$ such that $y \leq_{c} x$, the vector $x-y$ is also a vector in $\{0,1\}^{|E|}$.
Recursion (2) shows that if one know $Q_{1}(x)$ for all $x \in\{0,1\}^{|E|}$ then one can determine the probability configuration of any $m$-layer graph. However, as we have seen in Section 3.1, it is not easy to compute even $Q_{1}(x)$ for general $G$. Moreover, the number of terms in the summation in (2) is exponential in the graph size. Thus (2) is feasible only for very small graphs.

Note that (2) still requires exponential time even when the underlying graph has a simpler structure such as a tree. As we will see in the next two sections, recursions on the number of nodes lead to computationally more efficient algorithms for tree networks, although we do not know how to do it for general graphs.

\subsection{Links in Series}

Throughout this section, we assume that $q_{v}=q$ for all nodes $v$, $p_{\ell}=1$ for all links $\ell$, and $K=1$. The results can be extended to more general parameter settings, but the notation will be too complicated. When need arises, we can do computation using the more general algorithm in Section 4.3.

Consider the graph $G_{n}=\left(V_{n}, E_{n}\right)$ defined by $V_{n}=\{1,2, \ldots, n+$ $1\}$ and $E_{n}=\left\{e_{j}\right\}_{j=1}^{n}$, where $e_{j}=(j, j+1)$ for $j=1, \ldots, n$. In other words, $G_{n}$ is composed of $n+1$ nodes and $n$ links in series, $e_{1}, e_{2}, \ldots, e_{n}$; see Figure 2 .

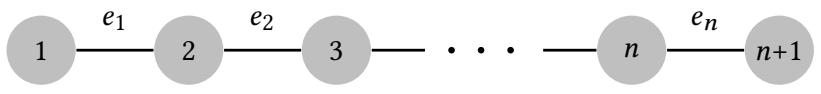

Figure 2: Line network $G_{n}$.

We are interested in calculating $Q_{G_{n}, x^{(n)}}$, the probability that links $e_{1}, \ldots, e_{n}$ are in state $x^{(n)}:=\left(x_{1}, \ldots, x_{n}\right) \in\{0,1\}^{n}$. Link $e_{j}$ is in state 1 (resp. state 0 ), denoted by $x_{j}=1$ (resp. $x_{j}=0$ ), if it is active (resp. inactive), namely, if nodes $j$ and $j+1$ belong to at least one common layer (resp. do not have any layer in common).

Here, $Q_{G_{n}, x^{(n)}}(m)$ will denote the probability that $G_{n}$ is in state $x^{(n)}$ given that node $n+1$ belongs to $m$ layers.

Since no confusion may occur, from now on we will drop the subscript $G_{n}$ in both $Q_{G_{n}, x^{(m)}}$ and $Q_{G_{n}, x^{(n)}}(m)$. With a slight abuse of notation, $0^{(n)}=(0, \ldots, 0)$ and $1^{(n)}=(1, \ldots, 1)$, where each vector has $n$ entries.

The following recursion holds for $Q_{x^{(n)}}(m), n \geq 2$,

$$
\begin{aligned}
& Q_{x^{(n)}}(m)=\bar{x}_{n} \bar{q}^{m} \sum_{i=0}^{M-m}\left(\begin{array}{c}
M-m \\
i
\end{array}\right) q^{i} \bar{q}^{M-m-i} Q_{x^{(n-1)}}(i) \\
& \quad+x_{n} \sum_{j=1}^{m}\left(\begin{array}{c}
m \\
j
\end{array}\right) q^{j} \bar{q}^{m-j} \sum_{i=0}^{M-m}\left(\begin{array}{c}
M-m \\
i
\end{array}\right) q^{i} \bar{q}^{M-m-i} \\
& \quad \times Q_{x^{(n-1)}}(i+j)
\end{aligned}
$$

with $\bar{q}=1-q, \bar{x}_{n}=1-x_{n}$, and $Q_{x^{(0)}}(\cdot)=1$ by convention. In particular,

$$
Q_{x^{(1)}}(m)=x_{1}\left(1-\bar{q}^{m}\right)+\bar{x}_{1} \bar{q}^{m} \text {. }
$$

The first term in the r.h.s. of (18) accounts for the fact that if link $e_{n}=(n, n+1)$ is in state $x_{n}=0$ then node $n$ cannot belong to the same layer as node $n+1$ (this occurs with probability $(1-q)^{m}$ as node $n+1$ belongs to $m$ layers) but otherwise can belong to any of the $M-m$ remaining layers, while the second term accounts for 
the fact that if link $e_{n}$ is in state $x_{n}=1$ then node $n$ needs to share at least one layer with node $n+1$ but otherwise can belong to any other layer(s).

We first calculate $Q_{0^{(n)}}(m)$, the probability that links $e_{1}, \ldots, e_{n}$ are all inactive given that node $n+1$ belongs to $m$ layer. This probability will turn out to be a key ingredient in the calculation of $Q_{x^{(n)}}$

Proposition 4.1 (Calculation of $Q_{0^{(n)}}(m)$ ). For any integer $m=0,1, \ldots, M, Q_{0^{(1)}}(m)=\bar{q}^{m}$, and $n \geq 2$,

$$
\begin{aligned}
Q_{0^{(n)}}(m)= & \bar{q}^{m}\left[1-(n-2) q^{2}+P_{n-1}(q)\right]^{m} \\
& \times\left[1-(n-1) q^{2}+P_{n}(q)\right]^{M-m},
\end{aligned}
$$

where $P_{1}, \ldots, P_{n}$ are polynomials in the variable $q$, recursively defined by

$$
P_{k}(q)=\bar{q} P_{k-1}(q)+q \bar{q} P_{k-2}(q)+q^{3}+(k-3) q^{4},
$$

for $k=3, \ldots, n$, with

$$
P_{1}(q)=P_{2}(q) \equiv 0 .
$$

Proof. For $n=1$, the conclusion follows from (4). For $n=2$, setting $x^{(2)}=0^{(2)}$ in (18) yields

$$
Q_{0^{(2)}}(m)=\bar{q}^{m} \sum_{i=0}^{M-m}\left(\begin{array}{c}
M-m \\
i
\end{array}\right) q^{i} \bar{q}^{M-m-i} Q_{0^{(1)}}(i) .
$$

Using $Q_{0^{(1)}}(i)=\bar{q}^{i}$, we obtain $Q_{0^{(3)}}(m)=\bar{q}^{m}\left(1-q^{2}\right)^{M-m}$, in agreement with (5).

We now use induction to complete the proof. Assume that (5) holds for $n=2, \ldots, n^{\prime}-1$ with $n^{\prime} \geq 3$, and let us show that it still holds for $n=n^{\prime}$. Setting $x_{1}, \ldots, x_{n^{\prime}-1}$ to zero in (18) and using the induction hypothesis and the binomial expansion, we obtain

$$
\begin{aligned}
Q_{0^{\left(n^{\prime}\right)}}( & m)=\bar{q}^{m}\left[1-\left(n^{\prime}-2\right) q^{2}+P_{n^{\prime}-1}(q)\right]^{m} \\
\times & {\left[1-\left(n^{\prime}-1\right) q^{2}+\bar{q} P_{n^{\prime}-1}(q)+q \bar{q} P_{n^{\prime}-2}(q)\right.} \\
& \left.+q^{3}+\left(n^{\prime}-3\right) q^{4}\right]^{M-m} \\
= & \bar{q}^{m}\left(1-\left(n^{\prime}-2\right) q^{2}+P_{n^{\prime}-1}(q)\right)^{m} \\
& \times\left[1-\left(n^{\prime}-1\right) q^{2}+P_{n^{\prime}}(q)\right]^{M-m}
\end{aligned}
$$

where the latter equality comes from the definition of $P_{n^{\prime}}(q)$. This completes the proof.

Corollary 4.2 (Calculation of $\left.Q_{0^{(n)}}\right)$. For any integer $n \geq 1$,

$$
Q_{0}(n)=\left[1-n q^{2}+P_{n+1}(q)\right]^{M} .
$$

The proof is straightforward by using Proposition 4.1 together with the identify

$$
Q_{0^{(n)}}=\sum_{m=0}^{M}\left(\begin{array}{l}
M \\
m
\end{array}\right) q^{m}(1-q)^{M-m} Q_{0^{(n)}}(m) .
$$

We are now in position to find $Q_{x^{(n)}}$, the probability that links $e_{1}, \ldots, e_{n}$ are in state $\left(x_{1}, \ldots, x_{n}\right)$. This result will be an easy consequence (see Proposition 4.4) of the next proposition that determines $Q_{x_{k}^{(n)}}(m)$, the probability that links $e_{k}, \ldots, e_{n}$ are in state $\left(x_{k}, \ldots, x_{n}\right)$ given that node $v_{k}$ belongs to $m$ layers, for $k=n, \ldots, 1$.

Proposition 4.3 (CAlculation of $Q_{x^{(n)}}(m)$ ). For any integer $n \geq 1, m=0,1, \ldots, M$,

$$
Q_{x^{(n)}}(m)=\sum_{j=0}^{n} h_{j} Q_{0^{(n-j)}}(m) \prod_{l=j+1}^{n}\left(\bar{x}_{l}-x_{l}\right)
$$

where $Q_{0^{(0)}}(\cdot) \equiv 1,\left\{Q_{0^{(j)}}(m)\right\}_{j=1}^{n-1}$ is given in (5), and $\left\{h_{j}\right\}_{j=0}^{n}$ are mappings depending on $x^{(n)}$ and $q$ recursively defined by

$$
\begin{aligned}
h_{j}= & x_{j} \sum_{r=0}^{j-1}\left(1-(j-1-r) q^{2}+P_{j-r}(q)\right)^{M} h_{r} \\
& \times \prod_{l=r+1}^{j-1}\left(\bar{x}_{l}-x_{l}\right)
\end{aligned}
$$

for $j=2, \ldots, n$, with

$$
h_{1}=x_{1} \text { and } h_{0}=1 \text {. }
$$

Proof. Letting $n=1$ in (9) and using (5), (11) and $Q_{0^{(0)}}(\cdot) \equiv 0$, gives (by convention $\prod_{l=1}^{0} \cdot=1$ )

$$
\begin{aligned}
Q_{x^{(1)}}(m) & =h_{0} Q_{0^{(1)}}(m)\left(\bar{x}_{1}-x_{1}\right)+h_{1} Q_{0^{(0)}}(m) \\
& =x_{1}\left(1-\bar{q}^{m}\right)+\bar{x}_{1} \bar{q}^{m},
\end{aligned}
$$

which is true by (4). Assume that (9) is true for $n=1, \ldots, n^{\prime}-1$. We show that it is still true for $n=n^{\prime}$.

From the induction assumption (9) with $n=n^{\prime}-1$, relation (47) and Lemma A.2 both given in Appendix A, we get

$$
\begin{aligned}
Q_{x^{\left(n^{\prime}\right)}}(m)=\bar{x}_{n^{\prime}} \bar{q}^{m} \sum_{j=0}^{n^{\prime}-1} h_{j} F\left(Q_{0^{n^{\prime}-1-j}}, m\right) \prod_{l=j}^{n^{\prime}-1}\left(\bar{x}_{l}-x_{l}\right) \\
+x_{n^{\prime}} \sum_{j=0}^{n^{\prime}-1} h_{j} G\left(Q_{0^{n^{\prime}-1-j}}, m\right) \prod_{l=j}^{n^{\prime}-1}\left(\bar{x}_{l}-x_{l}\right) \\
=\sum_{j=0}^{n^{\prime}-1} h_{j} Q_{0^{n^{\prime}-j}}(m) \prod_{l=j+1}^{n^{\prime}}\left(\bar{x}_{l}-x_{l}\right) \\
\quad+\sum_{j=0}^{n^{\prime}-1}\left(1-\left(m^{\prime}-1-j\right) q^{2}+P_{n^{\prime}-j}\right)^{M} h_{j} \prod_{l=j+1}^{n^{\prime}-1}\left(\bar{x}_{l}-x_{l}\right) \\
=\sum_{j=0}^{n^{\prime}-1} h_{j} Q_{0^{n^{\prime}-j}}(m) \prod_{l=j+1}^{n^{\prime}}\left(\bar{x}_{l}-x_{l}\right)+h_{n^{\prime}} \\
=\sum_{j=0}^{n^{\prime}} h_{j} Q_{0^{n^{\prime}-j}}(m) \prod_{l=j+1}^{n^{\prime}}\left(\bar{x}_{l}-x_{l}\right)
\end{aligned}
$$

where (12) follows from the definition of $h_{n^{\prime}}$ given in (10). This concludes the induction step.

Proposition 4.4 (CAlculation of $Q_{x^{(n)}}$ ).

For any integer $n \geq 1$,

$$
Q_{x^{(n)}}=\sum_{j=0}^{n} h_{j} Q_{0^{(n-j)}} \prod_{l=j+1}^{n}\left(\bar{x}_{l}-x_{l}\right)
$$

where $Q_{0^{(0)}}=1$, and $Q_{0^{(1)}} \ldots, Q_{0^{(n)}}$ are given in Corollary 4.2 and $h_{0}, \ldots, h_{n}$ are given in (10)-(11). 
Proof. The proof follows from the identity

$$
Q_{x^{(n)}}=\sum_{m=0}^{M}\left(\begin{array}{l}
M \\
m
\end{array}\right) q^{m} \bar{q}^{M-m} Q_{x^{(n)}}(m)
$$

together with Proposition 4.3 and (8).

We conclude this section by calculating the probability generating function (pgf) of the size of the connected component an arbitrary node belongs to, and the pgf of the number of active links.

For a path of length $n$ as represented in Fig. 2, let $C_{n}(i) \in$ $\{1, \ldots, n+1\}$ denote the size of the connected component that node $i$ belongs to, $i=1, \ldots, n$, and let $C_{n}(i, z)=\mathbb{E}\left[z^{C_{n}(i)}\right]$ denote the pgf for the distribution of $C_{n}(i)$. In particular, if node $i$ is inactive (i.e. does not belong to any layer) then $C_{n}(i)=1$. $C_{n}(i, z)$ satisfies the following recursion,

$$
C_{n}(i, z)=C_{i-1}(1, z) C_{n-i+1}(1, z) / z
$$

for $2 \leq i \leq n$ and $C_{n}(1)=C_{n}(n+1)$. As a result, one only needs to calculate $C_{n}(1, z)$ for any integer $n \geq 1$ to obtain $C_{n}(i, z)$. Henceforth, $C_{n}(1, z) \equiv C_{n}(z)$.

Define the vector $y^{(i)}$ of size $i \geq 2$ by $y^{(i)}=(1, \ldots, 1,0)$. We have (use (13))

$$
\begin{gathered}
C_{n}(z)=Q_{0^{(1)}} z+\sum_{i=2}^{n} z^{i} Q_{y^{(i)}}+z^{n+1} Q_{1^{(n)}} \\
=\quad Q_{0^{(1)}} z+\sum_{i=2}^{n} z^{i} \sum_{j=0}^{i-1}(-1)^{i-j-1} \hat{h}_{j} Q_{0^{(i-j)}} \\
+z^{n+1} \sum_{j=0}^{n}(-1)^{n-j} \hat{h}_{j} Q_{0^{(n-j)}}
\end{gathered}
$$

where for $j=1, \ldots, n$,

$$
\hat{h}_{j}=\sum_{r=0}^{j-1}(-1)^{j-r-1}\left(1-(j-1-r) q^{2}+P_{j-r}(q)\right)^{M} \hat{h}_{r},
$$

with $\hat{h}_{1}=\hat{h}_{0}=1$. From Corollary 4.2, $C_{n}(z)$ in (17) writes

$$
\begin{aligned}
C_{n}(z) & =\left(1-q^{2}\right)^{M} z+\sum_{i=2}^{n} z^{i} \sum_{j=0}^{i-1}(-1)^{i-j-1} \\
& \times\left(1-(i-j) q^{2}+P_{i-j+1}(q)\right)^{M} \hat{h}_{j} \\
& +z^{n+1} \sum_{j=0}^{n}(-1)^{n-j}\left(1-(n-j) q^{2}+P_{n-j+1}\right)^{M} \hat{h}_{j}
\end{aligned}
$$

where polynomials $P_{1}(q), \ldots, P_{n}(q)$ are recursively defined in (6)(7). Let $\bar{C}_{n}(i)$ be the expected size of the connected component node $i$ belongs to. From $\bar{C}_{n}(i)=d C_{n}(i, z) /\left.d z\right|_{z=1}$ and (14) we get

$$
\bar{C}_{n}(i)=\bar{C}_{i-1}(1)+\bar{C}_{n-i+1}(1)-1
$$

for $2 \leq i \leq n$ and $\bar{C}_{n}(1)=\bar{C}_{n}(n+1)$, where

$$
\begin{aligned}
\bar{C}_{n}(1) & =Q_{0^{(1)}}+\sum_{i=2}^{n} i \sum_{j=0}^{i-1}(-1)^{i-j-1} \hat{h}_{j} Q_{0^{(i-j)}} \\
& +(n+1) \sum_{j=0}^{n}(-1)^{n-j} \hat{h}_{j} Q_{0^{(n-j)}} .
\end{aligned}
$$

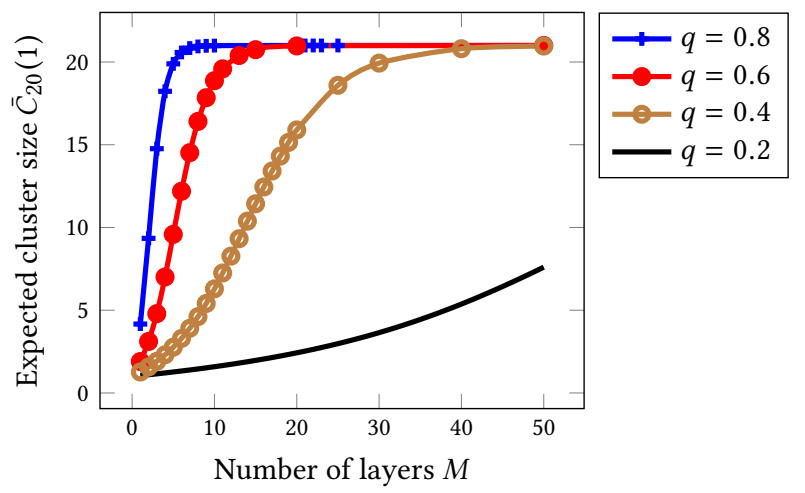

Figure 3: Expected size $\bar{C}_{20}(1)$ of the cluster containing node 1 in the line network of Figure 2 as a function of the number of layers $M$, for $n=20$ and $q \in\{0.2,0.4,0.6,0.8\}$.

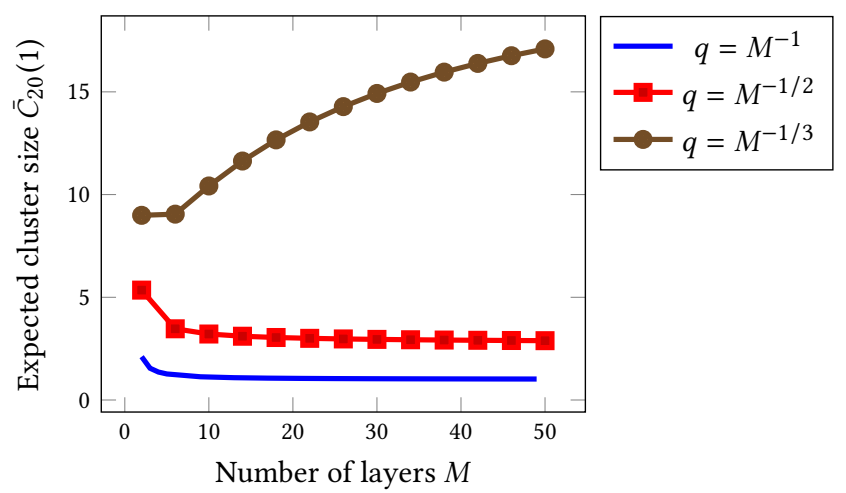

Figure 4: Expected size $\bar{C}_{20}(1)$ of the cluster containing node 1 in the line network of Figure 2 as a function of the number of layers $M$, for $n=20$ and $q \in\left\{M^{-1}, M^{-1 / 2}, M^{-1 / 3}\right\}$.

Let $L_{n}$ denote the number of active links in a line of length $n \geq 1$. Define $L_{n}(z)=\mathbb{E}\left[z^{L_{n}}\right]$ and $\bar{L}_{n}=\mathbb{E}\left[L_{n}\right] . L_{n}(z)$ is expressed as

$$
L_{n}(z)=\sum_{m=0}^{M} L_{n}^{(m)}(z), \quad n \geq 1,
$$

where $L_{n}^{(m)}(z)$ denotes the conditional active link pgf in a line of $n$ links given that node $n+1$ is active on exactly the first $m$ layers for $m=0, \ldots, M$. The following recursion holds for $L_{n}^{(m)}(z), n \geq 2$,

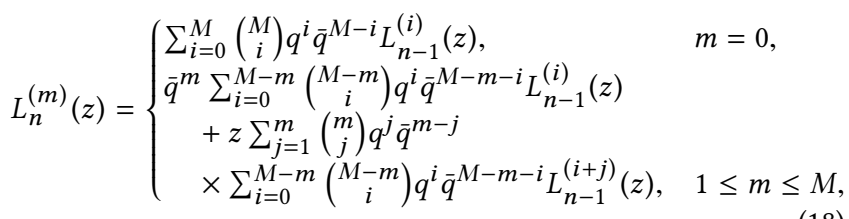

with $L_{1}^{(m)}(z)=\bar{q}^{m}+z\left(1-\bar{q}^{m}\right)$ for $m=0,1, \ldots, M$.

Figures 3 and 5 display the mappings $M \rightarrow \bar{C}_{n}(1)$ and $M \rightarrow \bar{L}_{n}$ for $n=20$, respectively, when $q \in\{0.2,0.4,0.6,0.8\}$. It shows the impact of having a finite number of layers on these metrics. Figures 4 and 6 investigate the behavior of these mappings when $q \in\left\{M^{-1}, M^{-1 / 2}, M^{-1 / 3}\right\}$. These plots show that both $\bar{C}_{n}(1)$ and $\bar{L}_{n}$ scale with $M$ as $q=1 / \sqrt{M}$. This result is rooted in the result 
that the limit of $1-\left(1-q^{2}\right)^{M}$ - the probability that a link is active - is non-zero as $M \uparrow \infty$ when $q=1 / \sqrt{M}$ (this limit is $1-e^{-1}$ ). The asymptotic behavior of a multilayer network as $M \rightarrow \infty$ is investigated in depth in Section 5.

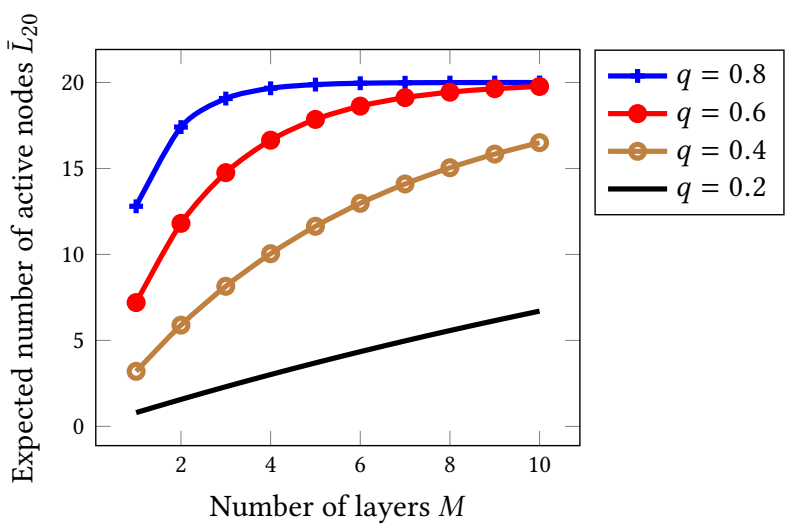

Figure 5: Expected number $\bar{L}_{20}$ of active nodes in a line network with 20 links as a function of the number of layers $M$, for $q \in$ $\{0.2,0.4,0.6,0.8\}$.

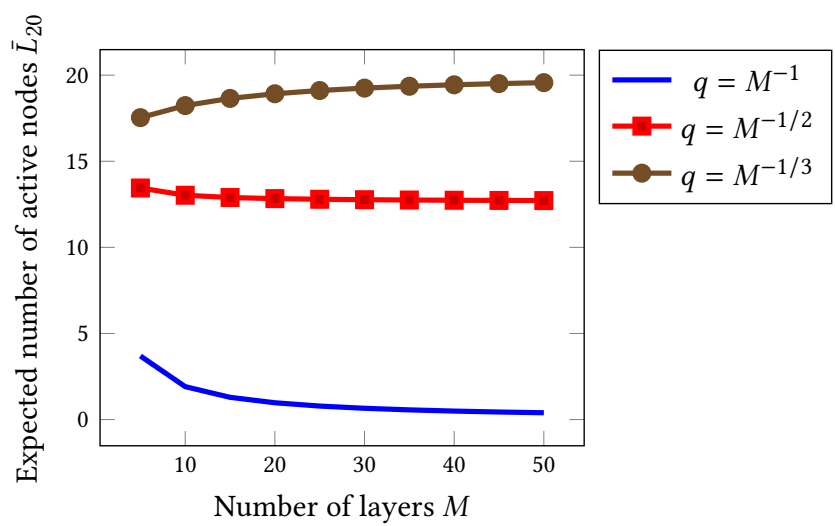

Figure 6: Expected number $\bar{L}_{20}$ of active nodes in a line network with 20 links as a function of the number of layers $M$, for $q \in$ $\left\{M^{-1}, M^{-1 / 2}, M^{-1 / 3}\right\}$.

\subsection{Recursion for Multilayer Trees}

In this section, we consider the case where the underlying graph is a tree $T$. We develop a recursion that provides a pseudo polynomial time algorithm to compute the probability of any link configuration. The recursion applies to all parameter settings of the model introduced in Section 2.

Pick any root $r$ for $T$. For $v \in V$, let $T_{v}$ denote the subtree rooted at $v$. With a slight abuse of notation, let $T_{v}(x)$ be the event that the links in $T_{v}$ are configured according to $x: E \rightarrow\{0,1\}$, i.e. $X_{\ell}=x_{\ell}$ for all $\ell \in T_{v}$. For $v, w \in V$, let $A_{v}$ be the number of layers on which $v$ is active, and $A_{v w}$ the number of layers on which both $v$ and $w$ are active. Note that $A_{v}$ has a binomial distribution with

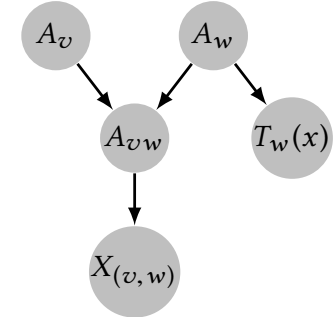

Figure 7: Dependency structure in the calculation of $g_{v w}^{(m)}$.

parameter $M$ and $q_{v}$, i.e.

$$
\mathbb{P}\left[A_{v}=m\right]=B\left(m ; M, q_{v}\right) \triangleq\left(\begin{array}{c}
M \\
m
\end{array}\right) q_{v}^{m}\left(1-q_{v}\right)^{M-m} .
$$

For $m=0,1, \ldots, M$, define $f_{v}^{(m)}(x)$ by

$$
f_{v}^{(m)}(x)=\mathbb{P}\left[A_{v}=m, T_{v}(x)\right] .
$$

Let $\operatorname{ch}(v)$ denote the set of $v$ 's children. Using the conditional independence of $X_{(v, w)}$ and $T_{w}(x)$ for different $w \in \operatorname{ch}(v)$, we obtain

$$
f_{v}^{(m)}(x)=\mathbb{P}\left[A_{v}=m\right] \prod_{w \in \operatorname{ch}(v)} g_{v w}^{(m)}(x)
$$

where

$$
g_{v w}^{(m)}(x)=\mathbb{P}\left[X_{(v, w)}=x_{(v, w)}, T_{w}(x) \mid A_{v}=m\right] .
$$

If $v$ is a leaf node, then $\operatorname{ch}(v)=\emptyset$ and $\prod_{w \in \operatorname{ch}(v)} g_{v w}^{(m)}(x)=1$ by convention.

To compute $g_{v w}^{(m)}(x)$, we make use of the dependency structure among the variables/events shown in Figure 7. Thus

$$
\begin{aligned}
g_{v w}^{(m)}(x)=\sum_{k=0}^{M} \sum_{j=0}^{\min \{m, k\}} & \mathbb{P}\left[A_{w}=k, T_{w}(x)\right] \\
& \times \mathbb{P}\left[A_{v w}=j \mid A_{v}=m, A_{w}=k\right] \\
& \times \mathbb{P}\left[X_{(v, w)}=x_{(v, w)} \mid A_{v w}=j\right] .
\end{aligned}
$$

The first factor in the summation is $f_{w}^{(m)}(x)$. By permutation symmetry, the second factor in the summation is given by a hypergeometric pmf, i.e.

$$
\mathbb{P}\left[A_{v w}=j \mid A_{v}=m, A_{w}=k\right]=H(j ; M, m, k) \triangleq \frac{\left(\begin{array}{c}
m \\
j
\end{array}\right)\left(\begin{array}{c}
M-m \\
k-j
\end{array}\right)}{\left(\begin{array}{c}
M \\
k
\end{array}\right)} .
$$

For the third factor, note that given $A_{v w}=j$, the number of layers on which link $(v, w)$ is active, $W_{(v, w)}$, has a binomial distribution with parameter $j$ and $p_{(v, w)}$. Thus

$$
\begin{aligned}
& \mathbb{P}\left[X_{(v, w)}=x_{(v, w)} \mid A_{v w}=j\right] \\
= & \left(1-x_{v w}\right)\left[1-\bar{F}_{B}\left(K ; j, p_{(v, w)}\right)\right]+x_{v w} \bar{F}_{B}\left(K ; j, p_{(v, w)}\right),
\end{aligned}
$$

where $\bar{F}_{B}\left(k ; j, p_{(v, w)}\right)=\sum_{\ell \geq k} B\left(\ell ; j, p_{(v, w)}\right)$ is the ccdf of binomial distribution with parameter $j$ and $p_{(v, w)}$.

We can compute $\left\{f_{v}^{(m)}(x): m=0,1, \ldots, M\right\}$ for all $v \in V$ sequentially from leaf nodes up to the root. The complexity is linear in $n=|V|$. The probability of configuration $x$ is then

$$
\mathbb{P}\left[X_{\ell}=x_{\ell}, \forall \ell \in E\right]=\sum_{m=0}^{M} f_{r}^{(m)}(x) .
$$


Since the pmfs of hypergeometric and binomial distributions can be computed in time polynomial in $M$, the above recursion can be computed in time $O(n \cdot \operatorname{poly}(M))$. Note that this pseudopolynomial time algorithm relies on the assumption that the layers are i.i.d. Although a similar recursion can be developed when layers are independent but have non-identical distributions, the complexity will scale as $M$ !, making it feasible only for small $M$.

Figure 9 displays the probability that all links are active in the star shaped network represented Figure 8 as a function of $q$, for different number of layers. Similarly, Figure 10 shows the probability that all links are active in a binary tree of depth 5 as a function of $q$, for different number of layers. These results were generated using the recursion discussed in this section with $q_{v}=q$ and $p_{\ell}=1$ for all nodes $v$ and links $l$.

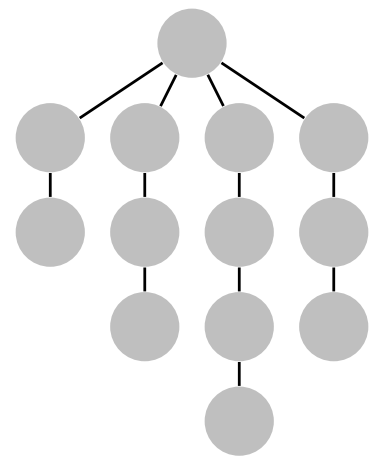

Figure 8: A star shaped network.

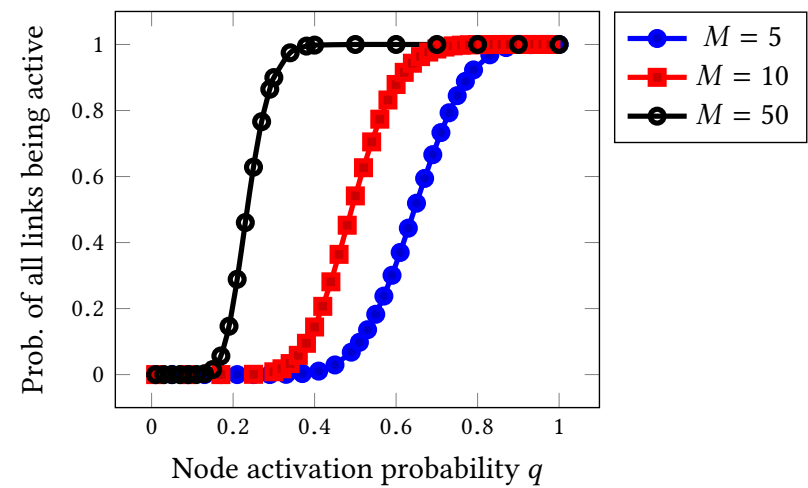

Figure 9: Probability that all links are active in the star shaped network in Figure 8 as a function of the node activation probability $q$.

\section{ASYMPTOTIC RESULTS}

In this section, we derive the link configuration distribution in the limit as the number of layers, $M$, goes to infinity and the probabilities $\left\{p_{\ell}\right\}_{\ell \in E}$ and $\left\{q_{i}\right\}_{i \in V}$ decreases as functions of $M$. This case is especially relevant when the multilayer network arises from snapshots of a single network as in duty-cycled wireless sensor networks, where $M$ can easily become very large. Even for moderate $M$, the asymptotics may already provide good approximations as shown in [1]. The main results are presented in Section 5.1 with proofs given in Section 5.2. The results are extended in Section 5.3 beyond the model of Section 2 to the case of non-identical layers.

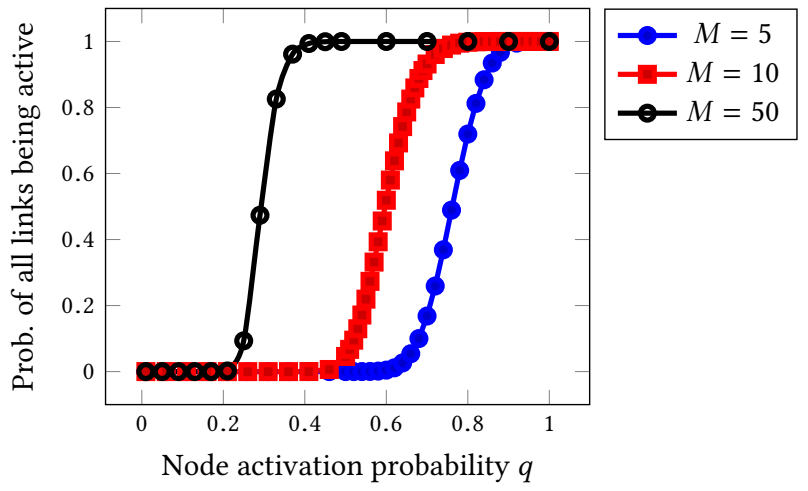

Figure 10: Probability that all links are active in a binary tree of height 5 as a function of the node activation probability $q$.

\subsection{Main Results}

Consider link $\ell=(i, j) \in E$. Note that its multiplicity $W_{\ell}$, the number of layers within which link $\ell$ is active (given in (1)) has a binomial distribution,

$$
\mathbb{P}\left[W_{\ell}=w\right]=\left(\begin{array}{l}
M \\
w
\end{array}\right)\left(p_{\ell} q_{i} q_{j}\right)^{w}\left(1-p_{\ell} q_{i} q_{j}\right)^{M-w}, \quad w=1, \ldots, M .
$$

If $M p_{\ell} q_{i} q_{j}$ has a finite positive limit as $M \rightarrow \infty$, a classical result shows that the above binomial distribution converges to a Poisson distribution. If we allow the natural interpretation of a Poisson distribution with rate parameter 0 or $\infty$ as a point mass at 0 or $\infty$, then we have the following,

Theorem 5.1. Suppose

$$
\lim _{M \rightarrow \infty} M p_{\ell} q_{i} q_{j}=\lambda_{\ell} \in[0, \infty]
$$

exists for link $\ell=(i, j)$. Then, as $M \rightarrow \infty$, the distribution of the multiplicity $W_{\ell}$ of $\ell$ converges to a Poisson distribution with parameter $\lambda_{\ell}$, i.e.

$$
\lim _{M \rightarrow \infty} \mathbb{P}\left[W_{\ell}=w\right]=\pi\left(w ; \lambda_{\ell}\right) \triangleq \frac{\lambda_{\ell}^{w}}{w !} e^{-\lambda_{\ell}} .
$$

The joint distribution of the $W_{\ell}$ 's may have a complicated correlation structure. However, when $p_{\ell}$ and $q_{i}$ scale with $M$ appropriately, the $W_{\ell}$ 's become asymptotically independent. We consider the case that $p_{\ell}$ and $q_{i}$ scale with $M$ as follows,

$$
\begin{aligned}
& p_{\ell} \sim c_{\ell} M^{-\alpha_{\ell}}, \quad \text { for } \ell \in E, \\
& q_{i} \sim d_{i} M^{-\beta_{i}}, \quad \text { for } i \in V,
\end{aligned}
$$

where $c_{\ell}, d_{i}>0$ and $\alpha_{\ell}, \beta_{i} \geq 0$. For a link $\ell=(i, j)$, the parameter $\lambda_{\ell}$ defined in (19) is then given by

$$
\lambda_{\ell}=\left\{\begin{array}{lll}
0, & \text { if } & \alpha_{\ell}+\beta_{i}+\beta_{j}>1 ; \\
c_{\ell} d_{i} d_{j}, & \text { if } & \alpha_{\ell}+\beta_{i}+\beta_{j}=1 ; \\
+\infty, & \text { if } & \alpha_{\ell}+\beta_{i}+\beta_{j}<1
\end{array}\right.
$$

For node $k$, let

$$
\mathcal{N}_{k}=\left\{i \in V:(k, i) \in E, \alpha_{(k, i)}+\beta_{k}+\beta_{i}=1\right\}
$$

We also assume the following condition,

$$
\beta_{k}<1 \text {, if }\left|\mathcal{N}_{k}\right| \geq 2 \text {. }
$$


Theorem 5.2. Under the conditions (20), (21) and (23), the collection of random variables $\left\{W_{\ell}: \ell \in E\right\}$ become asymptotically independent as $M \rightarrow \infty$, i.e. for any $\left\{w_{\ell}: \ell \in E\right\} \in \mathbb{N}^{E}$,

$$
\lim _{M \rightarrow \infty} \mathbb{P}\left[W_{\ell}=w_{\ell}, \forall \ell \in E\right]=\prod_{\ell \in E} \pi\left(w_{\ell} ; \lambda_{\ell}\right)=\prod_{\ell \in E} e^{-\lambda_{\ell}} \frac{\lambda_{\ell}^{w_{\ell}}}{w_{\ell} !},
$$

where $\lambda_{\ell}$ is given by (22).

Proof IDEA. In the large $M$ limit, each layer essentially has at most one link. Thus the configuration roughly follows a multinomial distribution, which in the limit becomes a product of Poisson distributions. The details are given in Section 5.2.

Note that condition (23) is necessary. The example below shows that asymptotic independence does not hold when condition (23) fails.

Example 5.3. Consider the line network in Figure 2 with three nodes. The probability that no links exist is given by

$$
\begin{aligned}
& \mathbb{P}\left[W_{(1,2)}=W_{(2,3)}=0\right] \\
= & {\left[1-q_{2}+q_{2}\left(1-q_{1} p_{(1,2)}\right)\left(1-q_{3} p_{(2,3)}\right)\right]^{M} . }
\end{aligned}
$$

If $\beta_{2}=1$ and $\alpha_{(1,2)}=\beta_{1}=\alpha_{(2,3)}=\beta_{3}=0$, then

$$
\begin{aligned}
& \lim _{M \rightarrow \infty} \mathbb{P}\left[W_{(1,2)}=W_{(2,3)}=0\right] \\
= & e^{-d_{2}+d_{2}\left(1-q_{1} p_{(1,2)}\right)\left(1-q_{3} p_{(2,3)}\right)} \\
= & \pi\left(0, \lambda_{(1,2)}\right) \pi\left(0, \lambda_{(2,3)}\right) e^{\lambda_{(1,2)} \lambda_{(2,3)} / d_{2}},
\end{aligned}
$$

which shows that $W_{(1,2)}$ and $W_{(2,3)}$ are not asymptotically independent.

It follows from Theorem 5.2 and the definition of $X_{\ell}$ that $\left\{X_{\ell}\right.$ : $\ell \in E\}$ is a set of asymptotically independent Bernoulli random variables with limiting marginal distribution $\lim _{M \rightarrow \infty} \mathbb{P}\left[X_{\ell}=1\right]=$ $\sum_{w \geq K} \pi\left(w ; \lambda_{\ell}\right)$. In particular, for $K=1$, the following corollary yields Theorem 1 of [1] if $G$ is a tree and the conjecture therein if $G$ is a general graph.

Corollary 5.4. Suppose $p_{\ell}=1$ for all $\ell \in E, q_{i}=d M^{-1 / 2}$ for all $i \in V$, and $K=1$. Then

$$
\lim _{M \rightarrow \infty} \mathbb{P}\left[X_{\ell}=x_{\ell}, \forall \ell \in E\right]=e^{-d^{2}(|E|-|\mathcal{E}|)}\left(1-e^{-d^{2}}\right)^{|\mathcal{E}|} .
$$

In the large $M$ limit, the merged network $\mathcal{G}$ has a giant component if $d$ exceeds the threshold $\sqrt{-\log \left(1-p_{c}\right)}$, where $p_{c}$ is the bondpercolation threshold of $G$.

Another consequence of Theorem 5.2 is the following trichotomy.

Corollary 5.5. Suppose $p_{\ell}=p \sim c M^{-\alpha}$ for all $\ell \in E, q_{i}=q \sim$ $d M^{-\beta}$ for all $i \in V$. Then in the limit $M \rightarrow \infty$, the network $\mathcal{G}$ is

(1) an empty network with no link, if $\alpha+2 \beta>1$;

(2) the entire network $G$, if $\alpha+2 \beta<1$;

(3) an Erdös-Rényi-like sub-network of $G$ where a link exists with probability $\sum_{w \geq K} \pi\left(w ; c d^{2}\right)$, if $\alpha+2 \beta=1$.

As an easy application of the results obtained in this section, consider the line network in Figure 2. When $q=1 / \sqrt{M}$ we know that links become independent as $M \rightarrow \infty$, with $p_{a}:=1-e^{-1}$ the (asymptotic) probability that a link is active. The expected size of the cluster node 1 belongs to, including this node, is then given by $\bar{C}_{a, n}=\left(1-p_{a}^{n+1}\right) /\left(1-p_{a}\right)$ and is plotted in Fig. 11 for $n=\{1, \ldots, 20\}$. We observe that $C_{a, n}$ converges fast w.r.t. $n$, the number of links. We have also plotted in this figure the mapping $n \rightarrow \bar{C}_{n}(1)$ for $q=1 / \sqrt{M}$ with $M=50$, which shows that making the assumption that links are independent when $q=1 / \sqrt{50}$ with $M=50$ yields a relative error of less then $10 \%$ across all values of $n \in\{1, \ldots, 20\}$. Similarly, the expected number of active links when $q=1 / \sqrt{M}$ and $M \rightarrow \infty$, given by $\bar{L}_{n, a}=n p_{a}$, is plotted in Fig. 12 as a function of $n$. We have also plotted in this figure the mapping $n \rightarrow \bar{L}_{n}$ (see Section 4.2) for $q=1 / \sqrt{M}$ with $M=40$ and $M=50$. For $M=40$ the relative error made by approximating $L_{n}$ by $\bar{L}_{n, a}$ does not exceed $14,1 \%$ across of all values of $n \in\{1, \ldots, 20\}$; it does not exceed $0.59 \%$ when $M=50$.

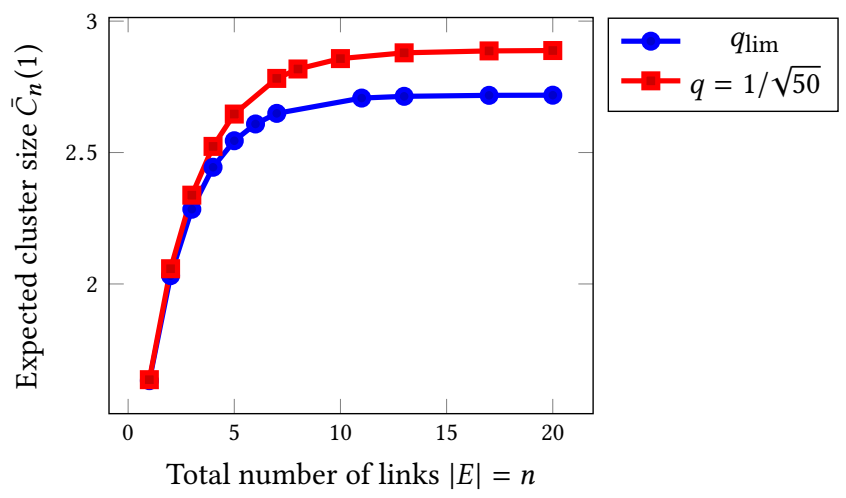

Figure 11: Expected size of cluster containing node 1 in the line network of Figure 2 as a function of the total number of links $n$, for $q=1 / \sqrt{M}$ and $M \rightarrow \infty\left(\right.$ refered to as $\left.q_{\mathrm{lim}}\right)$ and $q=1 / \sqrt{50}$.

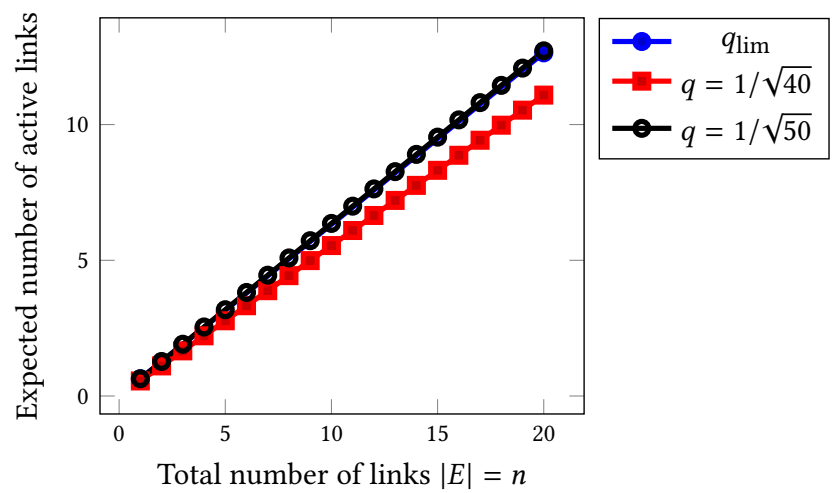

Figure 12: Expected number of active links in the line network of Figure 2 as a function of the total number of links $n$, for $q=1 / \sqrt{M}$ and $M \rightarrow \infty$ (refered to as $\left.q_{\lim }\right), q=1 / \sqrt{40}$, and $q=1 / \sqrt{50}$.

\subsection{Proof of Theorem $\mathbf{5 . 2}$}

We will use the following simple result, which holds for any probability measure.

Lemma 5.6. For any sequence of events $\left\{A_{M}\right\}$ and $\left\{B_{M}\right\}$ such that $\mathbb{P}\left[B_{M}\right] \rightarrow 1$ as $M \rightarrow \infty$, we have

$$
\mathbb{P}\left[A_{M}\right]-\mathbb{P}\left[A_{M} \cap B_{M}\right] \rightarrow 0, \quad \text { as } M \rightarrow \infty .
$$


Proof. The follows from the following inequalities,

$$
0 \leq \mathbb{P}\left[A_{M}\right]-\mathbb{P}\left[A_{M} \cap B_{M}\right] \leq \mathbb{P}\left[\overline{B_{M}}\right]=1-\mathbb{P}\left[B_{M}\right] .
$$

Since the limiting marginal distribution (19) degenerates to a point mass when $\lambda_{\ell}=0$ or $\infty$, by the above lemma, we only need to prove Theorem 5.2 for the case $\lambda_{\ell} \in(0, \infty)$ for all $\ell \in E$, or,

$$
\alpha_{\ell}+\beta_{i}+\beta_{j}=1, \quad \forall \ell=(i, j) \in E,
$$

which we assume throughout the rest of this section. Note that this assumption and (23) imply that $\beta_{i}<1$ for all $i \in V$ with degree greater than one in $G$. Let $\Gamma=\left\{i \in V: \beta_{i}<1\right\}$. If $\Gamma=\emptyset$, then every node has degree at most one, and hence (24) holds trivially. We henceforth assume $\Gamma \neq \emptyset$ and let

$$
\gamma=\min _{j: \in \Gamma}\left(1-\beta_{j}\right) \in(0,1] .
$$

We can also assume that there is no isolated node in $G$ since all such nodes can be removed.

Consider the $m$-th layer. Since the layers are i.i.d., the following analysis applies to all layers. Let $A_{\ell}$ be the event that link $\ell=(i, j)$ exists on this layer, i.e. $A_{\ell}=\left\{W_{m, \ell}=1\right\}$. The probability of this event is

$$
r_{\ell} \triangleq \mathbb{P}\left[A_{\ell}\right]=p_{\ell} q_{i} q_{j} \sim \lambda_{\ell} M^{-1} .
$$

Consider the probability that two links $\ell$ and $\ell^{\prime}$ co-exist on the $m$-th layer. There are two cases. If $\ell$ and $\ell^{\prime}$ do not share an endpoint, then $A_{\ell}$ and $A_{\ell^{\prime}}$ are independent, so the probability is

$$
\mathbb{P}\left[A_{\ell} \cap A_{\ell^{\prime}}\right]=\mathbb{P}\left[A_{\ell}\right] \mathbb{P}\left[A_{\ell^{\prime}}\right]=r_{\ell} r_{\ell^{\prime}} \sim \lambda_{\ell} \lambda_{\ell^{\prime}} M^{-2} .
$$

If $\ell$ and $\ell^{\prime}$ share an endpoint $i$, then $i \in \Gamma$ and $A_{\ell}$ and $A_{\ell}$ are conditionally independent given $i$. Thus

$$
\begin{aligned}
\mathbb{P}\left[A_{\ell} \cap A_{\ell^{\prime}}\right] & =\mathbb{P}\left[A_{\ell} \cap A_{\ell^{\prime}} \mid \text { node } i \text { active }\right] q_{i} \\
& =\mathbb{P}\left[A_{\ell} \mid \text { node } i \text { active }\right] \mathbb{P}\left[A_{\ell^{\prime}} \mid \text { node } i \text { active }\right] q_{i} \\
& =\frac{r_{\ell} r_{\ell^{\prime}}}{q_{i}} \sim \frac{\lambda_{\ell} \lambda_{\ell^{\prime}}}{d_{i}} M^{-2+\beta_{i}} \leq \frac{\lambda_{\ell} \lambda_{\ell^{\prime}}}{d_{i}} M^{-2+\gamma},
\end{aligned}
$$

where the last inequality follows from (27). In both cases, we have

$$
\mathbb{P}\left[A_{\ell} \cap A_{\ell^{\prime}}\right] \leq C M^{-1-\gamma},
$$

for some constant $C>0$ that does not depend on $\ell, \ell^{\prime}, m$ or $M$.

Let $\mu_{\ell}$ be the probability that $\ell$ is the only link on the $m$-th layer. We have

$$
\mu_{\ell}=\mathbb{P}\left[A_{\ell} \cap \bigcap_{\ell^{\prime} \in E: \ell^{\prime} \neq \ell} A_{\ell^{\prime}}^{c}\right] .
$$

From the identity

$$
A_{\ell}=\left(A_{\ell} \cap \bigcap_{\ell^{\prime} \in E: \ell^{\prime} \neq \ell} A_{\ell^{\prime}}^{c}\right) \cup\left(\bigcup_{\ell^{\prime} \in E: \ell^{\prime} \neq \ell} A_{\ell} \cap A_{\ell^{\prime}}\right),
$$

the union bound and (29), we find

$$
0 \leq \delta_{\ell} \triangleq r_{\ell}-\mu_{\ell} \leq \sum_{\ell^{\prime} \in E: \ell^{\prime} \neq \ell} \mathbb{P}\left[A_{\ell} \cap A_{\ell^{\prime}}\right] \leq|E| C M^{-1-\gamma} .
$$

Let $\mu_{0}$ be the probability that there is no link on the $m$-th layer, given by

$$
\mu_{0}=\mathbb{P}\left[A_{\ell}^{c}, \forall \ell \in E\right] .
$$

Bonferroni’s inequality

$$
\mathbb{P}\left(\bigcup_{\ell \in E} A_{\ell}\right) \geq \sum_{\ell \in E} \mathbb{P}\left(A_{\ell}\right)-\frac{1}{2} \sum_{\ell, \ell^{\prime} \in E, \ell \neq \ell^{\prime}} \mathbb{P}\left(A_{\ell} \cap A_{\ell^{\prime}}\right)
$$

and (29), yields

$0 \geq \delta_{0} \triangleq 1-\mu_{0}-\sum_{\ell \in E} r_{\ell} \geq-\frac{1}{2} \sum_{\ell \neq \ell^{\prime}} \mathbb{P}\left[A_{\ell} \cap A_{\ell^{\prime}}\right] \geq-\frac{|E|^{2}}{2} C M^{-1-\gamma}$.

Consider the event that $W_{\ell}=w_{\ell}$ for all $\ell \in E$ and each layer has at most one link. This event occurs if and only if each $\ell$ exists on a disjoint set of $w_{\ell}$ layers and the remaining $M-w$ layers are empty, where $w=\sum_{\ell \in E} w_{\ell}$. The corresponding probability is given by a multinomial distribution. Formally, if we let $B$ denote the event that each layer has at most one link, then

$$
\mathbb{P}\left[W_{\ell}=w_{\ell}, \forall \ell \in E, B\right]=\frac{M !}{(M-w) ! \prod \ell \in E w_{\ell} !} \mu_{0}^{M-w} \prod_{\ell \in E} \mu_{\ell}^{w_{\ell}},
$$

where $\mu_{\ell}$ and $\mu_{0}$ are defined in (30) and (32).

As $M \rightarrow \infty$,

$$
\frac{M !}{(M-w) !} \sim M^{w} .
$$

By (38), (31) and (33), we also have

$$
\mu_{\ell}^{w_{\ell}}=\left(r_{\ell}-\delta_{\ell}\right)^{w_{\ell}} \sim \frac{\lambda_{\ell}^{w_{\ell}}}{M^{w_{\ell}}},
$$

and

$$
\mu_{0}^{M-w}=\left(1-\sum_{\ell \in E} r_{\ell}-\delta_{0}\right)^{M-w} \rightarrow e^{-\sum_{\ell \in E} \lambda_{\ell}} .
$$

Plugging (35)-(37) into (34), we obtain as $M \rightarrow \infty$,

$$
\mathbb{P}\left[W_{\ell}=w_{\ell}, \forall \ell \in E, B\right] \rightarrow \prod_{\ell \in E} \frac{\lambda_{\ell}^{w_{\ell}}}{w_{\ell} !} e^{-\lambda_{\ell}},
$$

which is almost the same as the claim in (24). By Lemma 5.6, it suffices that $\mathbb{P}[B] \rightarrow 1$ as $M \rightarrow \infty$, which we show next.

Note that the probability that a given layer has at most one link is given by $\mu_{0}+\sum_{\ell \in E} \mu_{\ell}=1-\delta_{0}-\sum_{\ell \in E} \delta_{\ell}$. Since the layers are independent,

$$
\mathbb{P}[B]=\left(1-\delta_{0}-\sum_{\ell \in E} \delta_{\ell}\right)^{M} \sim e^{-M \delta_{0}-\sum_{\ell \in E} M \delta_{\ell}} \rightarrow 1,
$$

where we have used (31) and (33). This completes the proof of Theorem 5.2.

\subsection{Extension to Non-identical Layers}

So far we have assumed that different layers are i.i.d., i.e. $p_{\ell}=$ $\mathbb{E}\left[Y_{m, \ell}\right]$ and $q_{i}=\mathbb{E}\left[Z_{m, i}\right]$ for all layers $m \in \mathcal{M}, \ell \in E$ and $i \in V$. In this section, we will relax this assumption by letting $p_{m, \ell}=\mathbb{E}\left[Y_{m, \ell}\right]$ and $q_{m, i}=\mathbb{E}\left[Z_{m, i}\right]$. The development will parallel that for the i.i.d. case.

Recall the definition $W_{m, \ell}=Y_{m, \ell} Z_{m, i} Z_{m, j}$ for $\ell=(i, j)$. Link $\ell$ exists on the $m$-th layer if and only if $W_{m, \ell}=1$. Define

$$
r_{m, \ell} \triangleq \mathbb{E} W_{m, \ell}=p_{m, \ell} q_{m, i} q_{m, j} .
$$


We assume that as $M \rightarrow \infty$,

$$
\max _{1 \leq m \leq M} r_{m, \ell} \rightarrow 0
$$

and

$$
\mathbb{E} W_{\ell}=\sum_{m=1}^{M} r_{m, \ell} \rightarrow \lambda_{\ell} \in(0, \infty) .
$$

Then again a classical result (e.g. [19, Theorem 5.7]) shows that the marginal distribution of $W_{\ell}$ converges to a Poisson distribution with parameter $\lambda_{\ell}$ as in (19).

Let $R_{\ell, \ell^{\prime}}$ be the expected number of layers on which two links $\ell$ and $\ell^{\prime}$ co-exist, i.e.

$$
R_{\ell, \ell^{\prime}} \triangleq \sum_{m=1}^{M} \mathbb{E}\left[W_{m, \ell} W_{m, \ell^{\prime}}\right]
$$

We replace condition (23) by the following. If two links $\ell, \ell^{\prime} \in E$ share an endpoint, then as $M \rightarrow \infty$,

$$
R_{\ell, \ell^{\prime}} \rightarrow 0
$$

Note that in the i.i.d. case, (23) implies (42) as shown in the Section 5.2.

Theorem 5.7. Under the conditions (39), (40) and (42), the collection of random variables $\left\{W_{\ell}: \ell \in E\right\}$ become asymptotically independent as $M \rightarrow \infty$, i.e. (24) holds.

Proof. We first show that $R_{\ell, \ell^{\prime}} \rightarrow 0$ as $M \rightarrow \infty$ for any pair of links $\ell \neq \ell^{\prime}$. If $\ell$ and $\ell^{\prime}$ share an endpoint, this is assumption (42). If $\ell$ and $\ell^{\prime}$ do not share an endpoint, then $W_{m, \ell}$ and $W_{m, \ell^{\prime}}$ are independent, so

$$
R_{\ell, \ell^{\prime}}=\sum_{m=1}^{M} \mathbb{E} W_{m, \ell} \mathbb{E} W_{m, \ell^{\prime}}=\sum_{m=1}^{M} r_{m, \ell} r_{m, \ell^{\prime}} .
$$

By (39) and (40),

$$
R_{\ell, \ell^{\prime}}=\sum_{m=1}^{M} r_{m, \ell} r_{m, \ell^{\prime}} \leq \max _{1 \leq m^{\prime} \leq M} r_{m^{\prime}, \ell^{\prime}} \sum_{m=1}^{M} r_{m, \ell} \rightarrow 0,
$$

as $M \rightarrow \infty$. Thus $R_{\ell, \ell^{\prime}} \rightarrow 0$ as $M \rightarrow \infty$ for all $\ell \neq \ell^{\prime}$.

Let $\mu_{m, \ell}$ be the probability that $\ell$ is the only link on the $m$-th layer, By the same argument for (31) and (33), we obtain

$$
0 \leq r_{m, \ell}-\mu_{m, \ell} \leq \sum_{\ell^{\prime} \in E: \ell^{\prime} \neq \ell} \mathbb{E}\left[W_{m, \ell} W_{m, \ell^{\prime}}\right] .
$$

Summing over $m$,

$$
0 \leq \sum_{m=1}^{M} r_{m, \ell}-\sum_{m=1}^{M} \mu_{m, \ell} \leq \sum_{\ell^{\prime} \in E: \ell^{\prime} \neq \ell} R_{\ell, \ell^{\prime}}
$$

The last sum goes to zero as $M \rightarrow \infty$. Thus (39) and (40) imply $\mu_{m, \ell} \rightarrow 0$ and

$$
\sum_{m=1}^{M} \mu_{m, \ell} \rightarrow \lambda_{\ell}
$$

as $M \rightarrow \infty$
Let $\mu_{m, 0}$ the probability that there is no link on the $m$-th layer. Using first the same argument for (33) and then the argument above for $\mu_{m, \ell}$, we obtain $\mu_{m, 0} \rightarrow 0$ and

$$
\sum_{m=1}^{M}\left(1-\mu_{m, 0}\right) \rightarrow \sum_{\ell \in E} \sum_{m=1}^{M} r_{m, \ell}=\sum_{\ell \in E} \lambda_{\ell}
$$

as $M \rightarrow \infty$.

Now we compute the Laplace transform of the random variables $W_{\ell}, \ell \in E$. For $t=\left(t_{\ell}: \ell \in E\right)$ and $t_{\ell} \geq 0$, the Laplace transform $\phi(t)$ is given by

$$
\phi(t)=\mathbb{E} \exp \left(-\sum_{\ell \in E} t_{\ell} W_{\ell}\right) .
$$

Using (1) and the independence between layers, we obtain

$\phi(t)=\mathbb{E} \exp \left(-\sum_{\ell \in E} t_{\ell} \sum_{m \in \mathcal{M}} W_{m, \ell}\right)=\prod_{m \in \mathcal{M}} \mathbb{E} \exp \left(-\sum_{\ell \in E} t_{\ell} W_{m, \ell}\right)$.

For a given $m$,

$$
\mathbb{E} \exp \left(-\sum_{\ell \in E} t_{\ell} W_{m, \ell}\right) \geq \mu_{m, 0}+\sum_{\ell \in E} e^{-t_{\ell}} \mu_{m, \ell}
$$

and

$$
\mathbb{E} \exp \left(-\sum_{\ell \in E} t_{\ell} W_{m, \ell}\right) \leq 1-\sum_{\ell \in E} \mu_{m, \ell}+\sum_{\ell \in E} e^{-t_{\ell}} \mu_{m, \ell} .
$$

Using (43) and (44), we obtain

$\sum_{m=1}^{M}\left[1-\mathbb{E} \exp \left(-\sum_{\ell \in E} t_{\ell} W_{m, \ell}\right)\right] \rightarrow \sum_{\ell \in E} \lambda_{\ell}\left(1-e^{-t_{\ell}}\right), \quad$ as $M \rightarrow \infty$.

By Lemma 5.8 of [19], as $M \rightarrow \infty$,

$$
\phi(t) \rightarrow \exp \left(\sum_{\ell \in E} \lambda_{\ell}\left(e^{-t_{\ell}}-1\right)\right)=\prod_{\ell \in E} \exp \left(\lambda_{\ell}\left(e^{-t_{\ell}}-1\right)\right) .
$$

The limit is the product of the Laplace transforms Poisson distributions with parameters $\lambda_{\ell}$, which yields (24).

\section{RELATED WORK}

As discussed in the introduction of this paper, there has been an explosion of research in multilayer networks in recent years, mostly from the physics community; two recent review articles are [8, 20]. Multilayer networks have been applied to airline networks [14], transportation systems serving a common user population [15], many body problems arising in condensed matter physics [30], brain and neural networks [11] and scientific collaboration networks [6], in addition to those listed in the introduction. Various models of multilayer networks relevant to different application scenarios have been proposed. These have been used in studies of diffusion dynamics of multilayer networks [16], cascades [9, 41], spectral properties [36], robustness [12, 24, 31], failure mechanisms [13, 34], correlations [28], growing random multilayer networks [27], epidemic spread [22], community structure [25], and algorithmic complexity of finding short paths through co-evolving multilayer networks [5]. The connectivity properties of random multilayer networks have also been studied, such as the study of the properties 
of the giant connected component (GCC) in a random network with correlated multiplexity, i.e., where the node degree distributions across layers have positive (or negative) correlations [21].

Stochastic multilayer networks are those whose constructions can be described by one or more control parameters (such as probability of the presence of a node, edge or more complex attributes). For such networks, a wide variety of percolation formulations have been proposed and studied, e.g., competition between layers [40], weak percolation [7], $k$-core percolation [3], directed percolation [2], spanning connectivity of a multilayer site-percolated network [1], and bond percolation [17]. Our stochastic multilayer network model can be visualized as a layered extension of the classical site-bond percolation model $[18,39]$, where bonds and sites are independently occupied with probabilities $q$ and $p$.

The work closest to ours [1] considers a special case of our model where only node deactivations are permitted and these are characterized as i.i.d. Bernoulli random variables. This work focuses on deriving conditions under which the multilayer network percolates, i.e., identifying the deactivation probability threshold such that if the deactivation probability lies below this threshold, a single giant connected component emerges. [1] studied the percolation behavior as $M \rightarrow \infty$ and derived the threshold under the conjecture that links become asymptotically independent as $M \rightarrow \infty$ without proving that conjecture. We have established this conjecture to be true with Theorem 5.1 in Section 5. Moreover, much of our paper is concerned with characterizing and computing the multilayer network configuration distribution.

\section{CONCLUSIONS}

In this work, we introduced a new class of stochastic multilayer networks. Such a network is the aggregation of $M$ random subnetworks of an underlying connectivity graph $G$. This model finds applications in social networks and communication networks, and, more generally, in any scenario where a multilayer network is formed over a common set of nodes via coexisting means of connectivity. We showed that it is \#P hard to compute exactly and NP-hard to approximate link configuration probabilities for general $G$, and it remains NP-hard to compute these probabilities when $G$ is a clique. We derived efficient recursions for computing configuration probabilities when $G$ is a line or more generally a tree. We showed that for appropriate scalings of the node and link selection processes to a layer, link multiplicities have asymptotically independent Poisson distributions as $M$ goes to infinity.

\section{REFERENCES}

[1] Anonymous. 2016. Details omitted for double-blind reviewing. (2016)

[2] N Azimi-Tafreshi, SN Dorogovtsev, and JFF Mendes. 2014. Giant components in directed multiplex networks. Physical Review E 90, 5 (2014), 052809.

[3] N Azimi-Tafreshi, J Gómez-Gardenes, and SN Dorogovtsev. 2014. k- core percolation on multiplex networks. Physical Review E 90, 3 (2014), 032816.

[4] Amitabha Bagchi, Sainyam Galhotra, Tarun Mangla, and Cristina M Pinotti. 2015 Optimal radius for connectivity in duty-cycled wireless sensor networks. ACM Transactions on Sensor Networks (TOSN) 11, 2 (2015), 36.

[5] P. Basu, M. Dippel, and R. Sundaram. 2015. Multiplex networks: a Generative Model and Algorithmic Complexity. In Proc. IEEE/ACM International Conference on Advances in Social Networks Analysis and Mining (ASONAM) 2015.

[6] Federico Battiston, Jacopo Iacovacci, Vincenzo Nicosia, Ginestra Bianconi, and Vito Latora. 2016. Emergence of multiplex communities in collaboration networks. PloS one 11, 1 (2016), e0147451.

[7] Gareth J Baxter, Sergey N Dorogovtsev, José FF Mendes, and Davide Cellai. 2014 Weak percolation on multiplex networks. Physical Review E 89, 4 (2014), 042801.
[8] S. Boccaletti, G. Bianconi, R. Criado, C. I. del Genio, J. Gómez-Gardeñes, M. Romance, I. Sendiña-Nadal, Z. Wang, and M. Zanin. 2014. The structure and dynamics of multilayer networks. Physics Reports 544, 1 (2014), 1-122.

[9] C. D. Brummitt, K.-M. Lee, and K.-I. Goh. 2012. Multiplexity-facilitated cascades in networks. Phys. Rev. E 85 (2012), 045102.

[10] F. Buccafurri, V. D. Foti, G. Lax, A. Nocera, and D. Ursino. 2013. Bridge analysis in a social internetworking scenario. Inf. Sci. 224 (mar 2013), 1-18.

[11] Ed Bullmore and Olaf Sporns. 2009. Complex brain networks: graph theoretical analysis of structural and functional systems. Nature Reviews Neuroscience 10, 3 (2009).

[12] Davide Cellai, Eduardo López, Jie Zhou, James P. Gleeson, and Ginestra Bianconi. 2013. Percolation in multiplex networks with overlap. Phys. Rev. E 88 (Nov 2013), 052811. Issue 5. DOI : http://dx.doi.org/10.1103/PhysRevE.88.052811

[13] Manlio De Domenico, Albert Solé-Ribalta, Sergio Gómez, and Alex Arenas. 2014. Navigability of interconnected networks under random failures. Proceedings of the National Academy of Sciences 111, 23 (2014), 8351-8356.

[14] Wen-Bo Du, Xing-Lian Zhou, Oriol Lordan, Zhen Wang, Chen Zhao, and Yan-Bo Zhu. 2016. Analysis of the Chinese Airline Network as multi-layer networks. Transportation Research Part E: Logistics and Transportation Review 89 (2016), 108-116.

[15] Riccardo Gallotti and Marc Barthelemy. 2015. The multilayer temporal network of public transport in Great Britain. Scientific data 2 (2015).

[16] S. Gómez, A. Díaz-Guilera, J. Gómez-Gardeñes, C. J. Pérez-Vicente, Y. Moreno, and A. Arenas. 2013. Diffusion Dynamics on Multiplex Networks. Phys. Rev. Lett. 110 (Jan 2013), 028701. Issue 2. DOI : http://dx.doi.org/10.1103/PhysRevLett.110. 028701

[17] Adam Hackett, Davide Cellai, Sergio Gómez, Alexandre Arenas, and James P Gleeson. 2016. Bond percolation on multiplex networks. Physical Review X 6, 2 (2016), 021002 .

[18] J. M. Hammersley. 1980. A generalization of McDiarmid's theorem for mixed Bernoulli percolation. (001 007 1980). DOI: http://dx.doi.org/10.1017/ S0305004100057455

[19] Olav Kallenberg. 2006. Foundations of modern probability. Springer Science \& Business Media.

[20] M. Kivelä, A. Arenas, M. Barthelemy, J. P. Gleeson, Y. Moreno, and M. A. Porter. 2014. Multilayer Networks. Journal of Complex Networks 2 (2014), 203-271.

[21] Kyu-Min Lee, Jung Yeol Kim, Won kuk Cho, K-I Goh, and I-M Kim. 2012. Correlated multiplexity and connectivity of multiplex random networks. New fournal of Physics 14, 3 (2012), 033027. http://stacks.iop.org/1367-2630/14/i=3/a=033027

[22] V. Marceau, P-A. Noël, L. Hébert-Dufresne, A. Allard, and L-J. Dubé. 2011. Modeling the dynamical interaction between epidemics on overlay networks. Phys. Rev. E 84 (Aug 2011), 026105. Issue 2. DOI : http://dx.doi.org/10.1103/PhysRevE. 84.026105

[23] Giulia Menichetti, Daniel Remondini, Pietro Panzarasa, Raúl J Mondragón, and Ginestra Bianconi. 2014. Weighted multiplex networks. PloS one 9, 6 (2014), e97857.

[24] Byungjoon Min, Su Do Yi, Kyu-Min Lee, and K-I Goh. 2014. Network robustness of multiplex networks with interlayer degree correlations. Physical Review E 89, 4 (2014), 042811.

[25] Raul J Mondragon, Jacopo Iacovacci, and Ginestra Bianconi. 2017. Multilink Communities of Multiplex Networks. arXiv preprint arXiv:1706.09011 (2017)

[26] Y. Murase, J. Török, H-H. Jo, K. Kaski, and J. Kertész. 2014. Multilayer weighted social network model. Phys. Rev. E 90 (Nov 2014), 052810. Issue 5. DOI : http: //dx.doi.org/10.1103/PhysRevE.90.052810

[27] V. Nicosia, G. Bianconi, V. Latora, and M. Barthelemy. 2013. Growing Multiplex Networks. Phys. Rev. Lett. 111 (Jul 2013), 058701. Issue 5. DOI : http://dx.doi.org/ 10.1103/PhysRevLett.111.058701

[28] Vincenzo Nicosia and Vito Latora. 2015. Measuring and modeling correlations in multiplex networks. Physical Review E 92, 3 (2015), 032805.

[29] J. Orlin. 1977. Contentment in graph theory: covering graphs with cliques. In Indagationes Mathematicae (Proceedings), Vol. 80. Elsevier, 406-424.

[30] Alessandro Pelizzola. 1995. Critical temperature of two coupled Ising planes. Phys. Rev. B 51 (May 1995), 12005-12006. Issue 17. DOI : http://dx.doi.org/10.1103/ PhysRevB.51.12005

[31] Filippo Radicchi and Ginestra Bianconi. 2017. Redundant Interdependencies Boost the Robustness of Multiplex Networks. Phys. Rev. X 7 (Jan 2017), 011013. Issue 1. DOI : http://dx.doi.org/10.1103/PhysRevX.7.011013

[32] Jason Redi and Ram Ramanathan. 2011. The DARPA WNaN network architecture. In Military Communications Conference, 2011-milcom 2011. IEEE, 2258-2263.

[33] J. Redi Redi and R. Ramanathan. 2011. The DARPA WNaN Network Architecture. In Proceedings of the IEEE Military Communications (MILCOM) Conference. 22582263.

[34] Saulo DS Reis, Yanqing Hu, Andrés Babino, José S Andrade Jr, Santiago Canals, Mariano Sigman, and Hernán A Makse. 2014. Avoiding catastrophic failure in correlated networks of networks. arXiv preprint arXiv:1409.5510 (2014).

[35] D. Roth. 1996. On the hardness of approximate reasoning. Artificial Intelligence 82,1 (1996), 273-302. 
[36] A. Solé-Ribalta, M. De Domenico, N. E. Kouvaris, A. Díaz-Guilera, S. Gómez, and A. Arenas. 2013. Spectral properties of the Laplacian of multiplex networks. Phys. Rev. E 88 (Sep 2013), 032807. Issue 3. DOI : http://dx.doi.org/10.1103/PhysRevE.88. 032807

[37] Michael Szell, Renaud Lambiotte, and Stefan Thurner. 2010. Multirelational organization of large-scale social networks in an online world. Proceedings of the National Academy of Sciences 107, 31 (2010), 13636-13641.

[38] S. P. Vadhan. 2001. The complexity of counting in sparse, regular, and planar graphs. SIAM 7. Comput. 31, 2 (2001), 398-427.

[39] M. Yanuka and R. Englman. 1990. Bond-site percolation: empirical representation of critical probabilities. Fournal of Physics A: Mathematical and General 23, 7 (1990), L339. http://stacks.iop.org/0305-4470/23/i=7/a=010

[40] Kun Zhao and Ginestra Bianconi. 2013. Percolation on interdependent networks with a fraction of antagonistic interactions. Fournal of Statistical Physics 152, 6 (2013), 1069-1083.

[41] Di Zhou, Jianxi Gao, H. Eugene Stanley, and Shlomo Havlin. 2013. Percolation of partially interdependent scale-free networks. Phys. Rev. E 87 (May 2013), 052812 Issue 5.

\section{A APPENDIX}

Define

$$
\begin{aligned}
F(f, m)= & \sum_{i=0}^{M-m}\left(\begin{array}{c}
M-m \\
i
\end{array}\right) q^{i} \bar{q}^{M-m-i} f(i) \\
G(f, m)= & \sum_{i=0}^{M-m}\left(\begin{array}{c}
M-m \\
i
\end{array}\right) q^{i} \bar{q}^{M-m-i} \\
& \times \sum_{j=1}^{m}\left(\begin{array}{c}
m \\
j
\end{array}\right) q^{j} \bar{q}^{m-j} f(i+j) .
\end{aligned}
$$

LEMMA A.1. If $f(i)=a^{i} b^{M-i} c$ then

$$
\begin{aligned}
& F(f, m)=c b^{m}(q a+\bar{q} b)^{M-m} \\
& G(f, m)=c(q a+\bar{q} b)^{M}-c(q a+\bar{q} b)^{M-m}(\bar{q} b)^{m} .
\end{aligned}
$$

Proof. This follows directly from the binomial expansion.

Note that $Q_{x^{(k)}}(m)$ in (18) expresses as

$$
Q_{x^{(n)}}(m)=\bar{x}_{n} \bar{q}^{m} F\left(Q_{x^{(n-1)}}, m\right)+x_{n} G\left(Q_{x^{(n-1)}}, m\right) .
$$

LEMMA A.2.

$$
\begin{aligned}
& \bar{q}^{m} F\left(Q_{0^{(n-1)}}, m\right)=Q_{0^{(n)}}(m) \\
& G\left(Q_{0^{(n-1)}}, m\right)=\left(1-(n-1) q^{2}+P_{n}\right)^{M}-Q_{0^{(n)}}(m) .
\end{aligned}
$$

Proof. (48) follows from (47) with $x_{1}=\cdots=x_{n}=0$; (49) is easily obtained by using (5), (6) and Lemma A.1. 